\title{
Effectiveness of muscle basal lamina carrying neural stem cells and olfactory ensheathing cells in spinal cord repair
}

\author{
X.W. Kang ${ }^{1}$, J.L. Hu ${ }^{2}$, S.K. Wang ${ }^{1}$ and J. Wang ${ }^{1}$ \\ ${ }^{1}$ Department of Orthopedics, Second Hospital of Lanzhou University, \\ Lan Zhou, China \\ ${ }^{2}$ Department of Orthopedics, The First Hospital of Baiyin City, Bansu, China \\ Corresponding authors: X.W. Kang / J.L. Hu \\ E-mail: kangxuewen_kxw@163.com / hujieliang_1@163.com
}

Genet. Mol. Res. 14 (4): 13437-13455 (2015)

Received January 7, 2015

Accepted May 25, 2015

Published October 28, 2015

DOI http://dx.doi.org/10.4238/2015.October.28.5

\begin{abstract}
We examined the effect of muscle basal lamina (MBL) with neural stem cells (NSCs) and olfactory ensheathing cells (OECs) on spinal cord injury repair. Seventy-two Sprague-Dawley rats were subjected to spinal cord hemisection and divided into 6 groups. In blank control group (group A), the ends of the spinal cord hemisection model were flushed with physiological saline. In NSC transplantation group (B), OEC transplantation group (C), MBL with NSC transplantation group (D), MBL with OEC transplantation group (E), and MBL with NSC and OEC transplantation group (F), NSCs, OECs, MBL with NSCs, MBL with OECs, and MBL with NSCs and OECs were implanted into the ends of the hemisection model. Survival and migration of transplanted cells were detected by immunohistochemistry and immunofluorescence after 4 and 8 weeks. Hind limb function repair was evaluated by Bundle branch block score at various time points before and after surgery. MBL could promote NSC growth along its lumen and promote host cell advancement in the lumen, reducing local inflammatory responses. Using MBL with NSCs
\end{abstract}


and/or OECs for spinal cord repair shows advantages over simple cell transplantation. Group F contained more nerve cells in muscle basal lamina than group E. This method is useful for forming more axons, synaptic connections, and signal transduction pathways. However, these new axons showed nerve demyelination, which may greatly limit nerve signal conduction. In group F, OECs could induce neural stem cells, axonal growth, and synaptic connection formation, but its role is limited.

Key words: Muscle basal lamina; Olfactory ensheathing cells; Neural stem cells; Spinal cord injury; Transplantation

\section{INTRODUCTION}

In recent years, many studies have reported the repair of spinal cord injury (SCI) by translation of neural stem cells (NSCs), olfactory ensheathing cells (OECs), or other cells (Boido et al., 2011; Amr et al., 2014; Binan et al., 2014). However, because of the barrier function of glial scar, limited effects were demonstrated using such methods (Binan et al. 2014). Muscle basal lamina (MBL), a new scaffold material, can cross the glial scar barrier, bridging the cavity and providing axon guidance; thus, MBL is considered to be an effective tool for overcoming the difficulties caused by glial scars (Fansa et al., 2002). In this study, we grafted MBL carrying NSCs and OECs into the SCI site to take advantage of the ability of NSCs to differentiate into neurons to fill the cavity, as well as the function of OECs to provide and store nutritional molecules for the regenerated nerve and inhibit glial proliferation, scar formation, and myelinization. We hypothesized that this method would improve SCI repair. Therefore, in this study, we created this complex and employed both morphologic and functional examination to determine whether the complex could facilitate the repair process, as well as explore the mechanism.

\section{MATERIAL AND METHODS}

\section{Animals, reagents, and culture media}

Sprague-Dawley (SD) rats were used in this study. Male and female rats weighing 200-250 g were supplied by the Animal Experimental Center of Gansu College of Traditional Chinese Medicine. Details regarding the reagents and culture media are as follows: special culture media for rat NSCs (containing 1:1 DMEM/F12, 2\% B27, $20 \mathrm{ng} / \mathrm{mL}$ basic fibroblast growth factor, $20 \mathrm{ng} / \mathrm{mL}$ epidermal growth factor, and $100 \mu \mathrm{g} / \mathrm{mL}$ penicillin, $100 \mu \mathrm{g} / \mathrm{mL}$ streptomycin; Gibco, Grand Island, NY, USA); nestin antibody (rabbit-anti-rat antibody); 5-bromodeoxyuridine (BrdU); primary antibodies (rabbit-anti-rat antibody; Gibco); cell culture media; $10 \%$ fetal bovine serum (Sigma, St. Louis, MO, USA); 0.25\% pancreatin (Sigma); P75 antibodies (rabbit-anti-rat antibody); fluorescein isothiocyanate (FITC) secondary antibodies (goat-anti-rabbit antibody); Hochest33342; neuron-specific enolase (NSE; Sigma); and Immunohistochemistry Kit (Maixin-Bio, Ultra-Sensitive SP, KIT-9701, DouSPTM, KiT-9999). 


\section{Isolation, culture, and identification of NSCs}

Embryonic SD rats at a gestational age of 14 weeks were selected. After peeling off the embryonic membrane, we removed the brain and then isolated meninges and vessels under a microscope (CX22, Olympus Corporation), while the diencephalon was preserved. The tissue was sectioned, placed in phosphate-buffered saline, and resuspended. This mixture was made into a single-cell suspension using a 200-mesh cell screen. Culture media for rat NSCs was added, the cells were counted, and the cell concentration was adjusted to $1.0 \times 10^{6} / \mathrm{mL}$. The cells were added to cell culture bottles and incubated at $5 \% \mathrm{CO}_{2}$ and $37^{\circ} \mathrm{C}$ (Thermo HERAcell 150i/240i, Thermo Fisher Scientific Inc.). The culture medium was changed every 3 days; the suspension was placed in a disposable centrifuge tube and centrifuged for $5 \mathrm{~min}$ at $1000 \mathrm{~g}$. The supernatant was discarded, culture media was added to the precipitate to a total volume of 5 $\mathrm{mL}$, and cells were grown in cell culture bottles. Approximately 7 days later, the neurosphere diameter reached $100 \mu \mathrm{m}$, indicating that subculture could be conducted. We first centrifuged the suspension as described above and changed the supernatant. Second, we added 0.5-1 mL culture media and separation medium. After 5 min incubation at $37^{\circ} \mathrm{C}$, we prepared the singlecell neurosphere suspension by suspending gently. Finally, we added culture media, counted the cells, readjusted the cell concentration to $1.0 \times 10^{6} / \mathrm{mL}$, and incubated the cells. Thirdgeneration cells were attached to slides coated with poly-L-lysine for immunofluorescence (TriM Scop, LaVision BioTec GmbH, Germany) analysis of the NSC-specific antigen nestin. The primary antibody was 1:200 rabbit-anti-rat nestin and the secondary antibody was 1:100 FITC goat-anti-rabbit fluorescent antibody.

\section{Isolation, culture, and identification of OECs}

After killing selected rats, we opened the skull, isolated the olfactory bulb, and peeled meninges and vessels under a microscope. The olfactory bulb was sectioned within $\mathrm{DMEM} / \mathrm{F} 12$ and a cell suspension was prepared. Next, $0.25 \%$ trypsinization was conducted for $5 \mathrm{~min}$, which was terminated by adding $10 \%$ fetal calf serum. The solution was filtered through a 200 -mesh cell screen and centrifuged for $3 \mathrm{~min}$ at $1000 \mathrm{r} / \mathrm{min}$; the supernatant was discarded. Culture medium was added to the suspension for single-cell resuspension and the concentration was adjusted to $1.0 \times 10^{6} / \mathrm{mL}$. Cells were placed into cell culture bottles for differential attachment culture and isolation. During this step, the bottle was switched each day, during which the adherent cells were discarded and the suspended cells were further cultured. Two days later, we added AraC to suppress fibroblast growth; cell purity reached over $90 \%$ by the 7 th day, which was typical for cell culture and OEC identification. Wells showing the highest purity were used for further analysis. Cells were attached to slides coated with poly-L-lysine for immunofluorescence analysis. A 1:100 dilution of rabbitanti-rat NGFR p75 was the primary antibody and 1:100 goat-anti-rabbit FITC fluorescent antibody was the secondary used for OECs. Specific operation was according to the relevant instructions.

\section{Preparation and identification of MBL}

SD rats of either gender weighing 200-250 g were examined. Rats were cut after intraperitoneal injection with $0.4 \%$ pentobarbital sodium for anesthetization. Next, the 
bilateral paravertebral muscles of the rats were extracted and cut into $2 \times 2 \times 1 \mathrm{~cm}$ strips. Muscle strips were placed in ultrapure water and shaken for $48 \mathrm{~h}$ in incubator shakers at $50 \mathrm{rpm}$ and $37^{\circ} \mathrm{C}$. Next, they were switched into pure Triton X-100 solution and shaken for $48 \mathrm{~h}$ again under the same conditions; samples were placed in $1 \%$ sodium dodecyl sulfate, shaken for another $48 \mathrm{~h}$, placed in ultrapure water, shaken for $24 \mathrm{~h}$, placed in phosphatebuffered saline, and shaken for another $24 \mathrm{~h}$. The muscle strips were cut into $2 \times 2 \times 1$ $\mathrm{cm}^{3}$ sections and stored at $-20^{\circ} \mathrm{C}$ until use. Some strips were used for identification. These samples were fixed in $4 \%$ paraformaldehyde for $12 \mathrm{~h}$, followed by pathological sectioning and hematoxylin and eosin staining. Finally, we observed the morphological changes of MBL under a microscope.

\section{Labeling NSCs and the verifying}

We selected the well-growing 3rd generations. After adding $15 \mu \mathrm{M}$ BrdU to the previous culture medium, the cultures were incubated overnight at $37^{\circ} \mathrm{C}$. Some the labeled cells were placed on a 6-well plate to facilitate attachment onto the slides coated with poly-L-lysine, which were used to evaluate the effectiveness of cellular labeling using an immunofluorescence test.

\section{In vitro culture and identification of complex combining NSCs and MBL}

The labeled NSC suspension was centrifuged and prepared as a single-cell suspension after discarding the supernatant. The prepared suspension was then injected into disinfected MBL using a $1-\mathrm{mL}$ fine-needle injector. For disinfection, MBL were soaked into $75 \%$ alcohol overnight and washed with sterile phosphate-buffered saline. MBL was completely submerged in the media on a 6-well plate for routine culture. Some of the complex was used for hematoxylin and eosin, immunohistochemistry, and immunofluorescence staining on the 3rd, 5th, and 7th days for identification. For immunohistochemistry staining, 1:200 rabbitanti-rat BrdU was used as the primary antibody and goat-anti-rabbit biotin-labeled as the secondary antibody. For immunohistochemistry staining, 1:200 rabbit-anti-rat BrdU was used as the primary antibody, while the secondary was 1:100 FITC goat-anti-rabbit fluorescent antibody. All operations were conducted based on the instructions.

\section{Labeling OECs with Hochest33342}

Similarly, to well-growing OECs, $10 \mu \mathrm{M}$ Hochest33342 was added to the culture medium and incubated for $4 \mathrm{~h}$ at $37^{\circ} \mathrm{C}$.

\section{In vitro culture and identification of complex of OECs and MBL}

The combination procedure was the same as that used for NECs and MBL. The complex was cultured and tested on the $3 \mathrm{rd}$, 5th, and 8th days by immunofluorescence staining to evaluate OEC proliferation in MBL. A 6- $\mu \mathrm{m}$ frozen section of the complex was prepared. After patching and mounting with glycerin, samples were observed under ultraviolet radiation directly with a microscope. Cells displaying blue fluorescence were OECs. 


\section{In vitro culture and identification of complex of NSCs, OECs, and MBL}

First, we performed the injection and combination of the 3 cell types using the method described above. Second, the samples were placed on a 6-well plate for routine culture (completely submerged in the media). On the $3 \mathrm{rd}$, 5th, and 7 th days, the complex was identified by hematoxylin and eosin, immunohistochemistry, and immunofluorescence staining.

\section{Grouping, hemisection SCI modeling, and transplantation}

Seventy-two rats from the same litter was selected and grouped into a blank control group (group A), NSCs only group (group B), OECs only group (group C), NSCs + MBL group (group D), OECs + MBL group (group E), and NSCs + OECs + MBL group (group F) using a randomized digital table. Each group included 12 rats. Rats were anesthetized by intraperitoneal injection of $0.4 \%$ pentobarbital sodium and cut layer-by-layer along the dorsal median line. We opened the spinous process and vertebral plate of T10 to expose the spinal dura mater, followed by a longitudinal incision. Next, we cut through the right spinal cord using an iridectome and cut out $1 \mathrm{~mm}$ long spinal cord from the ends. For rats in group A, incisions were closed layer-by-layer after washing with normal saline; in groups $\mathrm{B}$ and $\mathrm{C}$, we injected $1.0 \times 10^{6} / \mathrm{mL}$ of the corresponding suspension into the injured spinal cord site using a fine needle injector after closing the dura mater, and then sewed the cut. In groups D, E, and $\mathrm{F}$, the $1 \times 1 \times 1 \mathrm{~mm}$ complex were placed in the injured, and the dura matter and incision were then closed. One week later, we injected penicillin once per day and conducted bladder massaging twice (once in the morning and once in the evening) each day for urination.

\section{Assessment of locomotor function and morphological changes}

Locomotor function was assessed and scored according to the Basso, Beattie, and Bresnahan rank scale (BBB). On the day before transplantation and on the 2nd, 4th, and 8th days after transplantation, the recovery of locomotor function of rats' hind legs were assessed independently by 2 persons using an improved method. We calculated the average of the results. Morphological changes were observed using pathological sections. Four rats were selected from each group at the 4th and 8th weeks after the surgery. Their spinal cords were fixed by heart perfusion using paraformaldehyde, removed, and soaked in $30 \%$ sucrose solution overnight. Next, all spinal cords were stained by immunohistochemistry and immunofluorescence for later observation under microscopes. Specially, spinal cords in group $\mathrm{F}$ were stained with double- immunohistochemistry using the DouSPTM Kit; staining was conducted according to the manufacturer instructions. Staining was evaluated as follows. For "BrdU + P75", BrdU-positive cells were located in the nucleus and were black-blue; P75-positive staining cells were located in the cytoplasm or membranes and were red in color. For the "NSE + BrdU", NSE-positive cells were located in the cytoplasm or membranes and were red in color; BrdU-positive cells were located in the nucleus and were black-blue. Electron microscopy observation was conducted for 1 rat from group $\mathrm{F}$ after the spinal cord was fixed through heart perfusion using glutaraldehyde and then soaked in glutaraldehyde overnight. Samples were observed by transmission electron microscopy and scanning electron microscopy. 


\section{Statistical analysis}

Statistical analysis was performed using SPSS version 17.0 (SPSS, Inc., Chicago, IL, USA). BBB scores are reported as means \pm standard deviation. The $t$-test was applied for intergroup comparison. Differences were considered to be statistically significant when $\mathrm{P}<0.05$.

\section{RESULTS}

\section{Primary culture and identification of NSCs}

After culture for 7 days, primary NSCs formed into a neural sphere with a diameter of nearly $100 \mu \mathrm{m}$, which consisted of tens to hundreds of cells. The sphere showed no obvious bulge and good refractivity (Figure 1). The results of immunofluorescence reveled that primary cultured NSCs in our study were nestin-positive (Figure 2A-B).

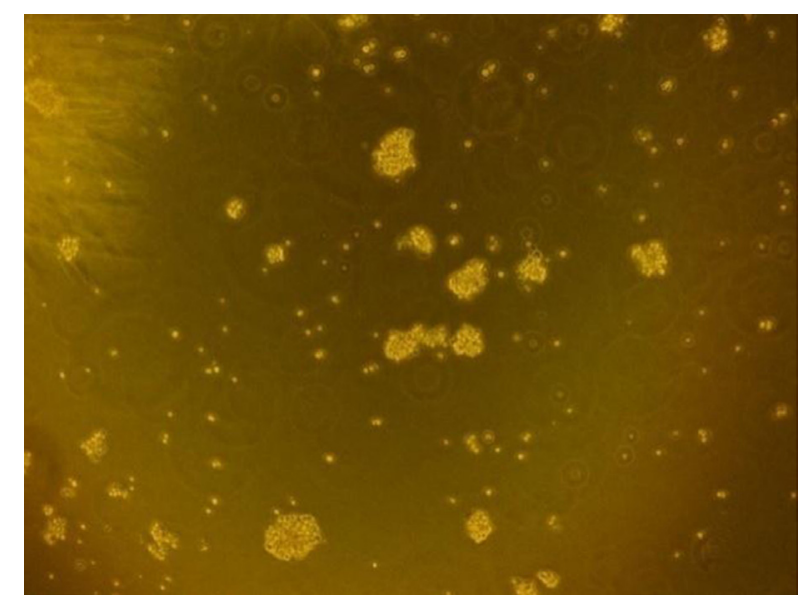

Figure 1. Primary cultured NSCs for 7 days (10X).
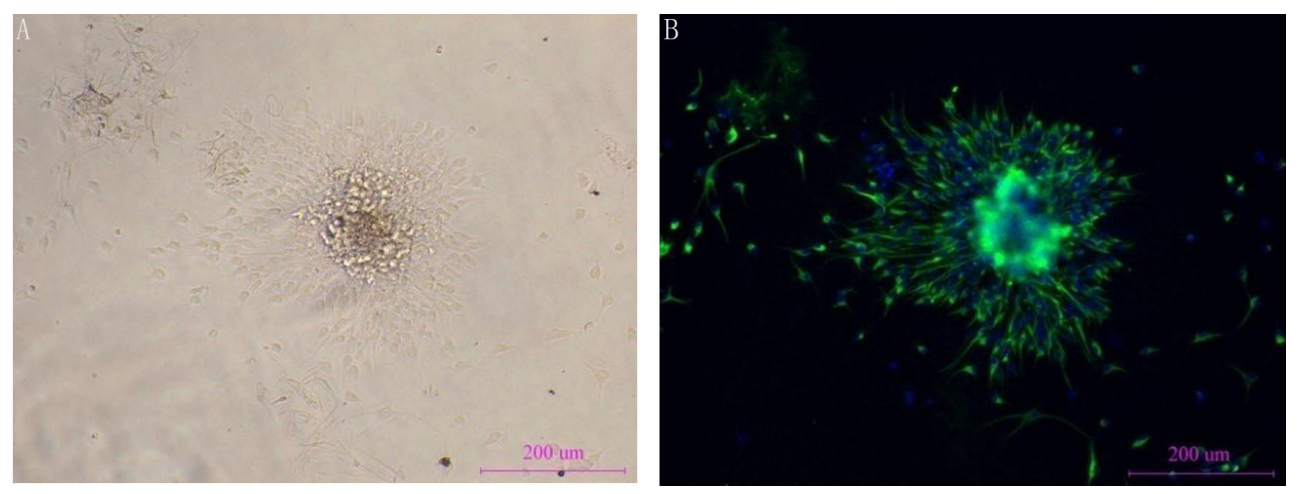

Figure 2. A. Bright field (10X). B. Identified using immunofluorescence based on ultraviolet light with primary anti-nestin, secondary antibody: FITC (green fluorescent), DAPI staining of nuclei, blue fluorescence, $200 \mu \mathrm{m}$. 


\section{Primary culture and identification of OECs}

An increase in primary OECs was observed on the 3rd day. Most cells developed small neurites and bipolar cells were occasionally observed. On the 4th day, bipolar cells with thin and outstretched neuritis were found, while few multipolar cells were observed. On the 7th day, adherent bipolar and multipolar cells were observed to be reticular and NGFR p75positive (Figure 3).

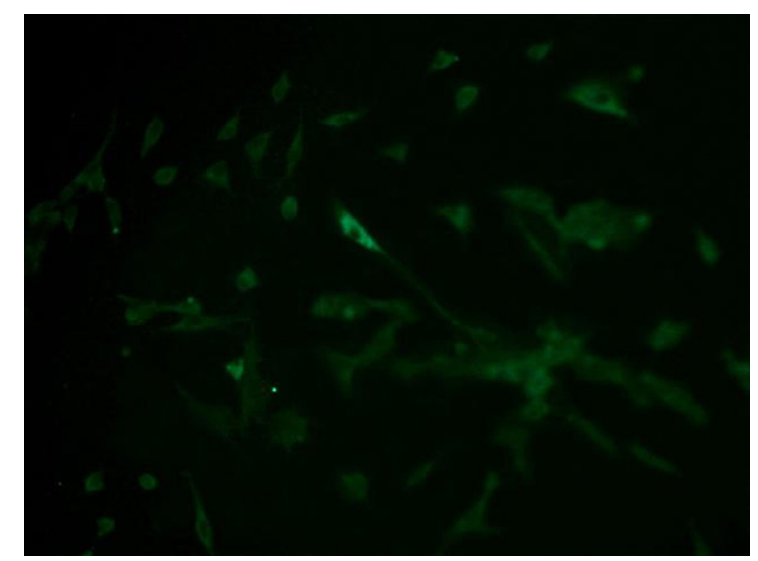

Figure 3. Primary OEC identification using immunofluorescence based on P75 (40X) (Blu-ray excitation).

\section{Evaluation of MBL}

Microscopically, orderly arranged and equally eosinophilic basement membrane components were observed, while muscle cells were not present. Tiny lumens with a diameter in the micron range were viewed between adjacent basement membranes, which may serve as channels for the migration and adhesion of transplanted cells (Figure 4).

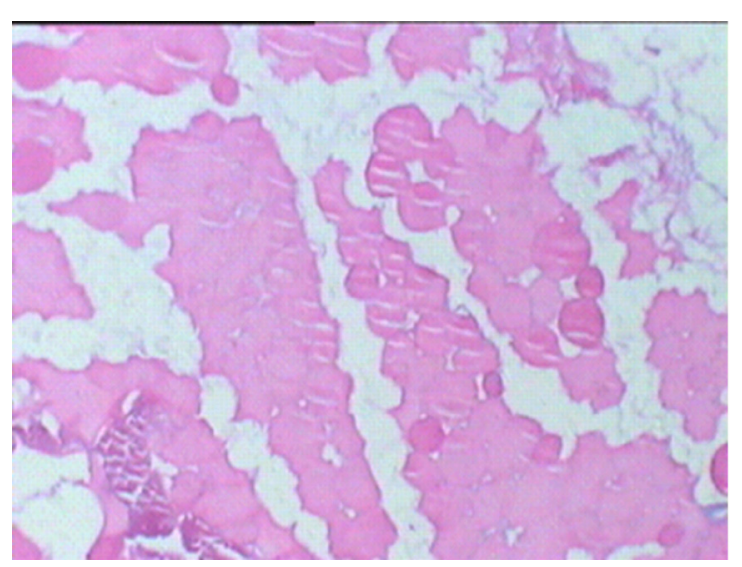

Figure 4. MBL hematoxylin and eosin staining (40X). 


\section{Examination of NSCs labeled with BrdU}

The nucleus showed green fluorescence and was round or oval. Nuclei also had long and thin neuritis and good refractivities, and more than 95\% were BrdU-positive (Figure 5).

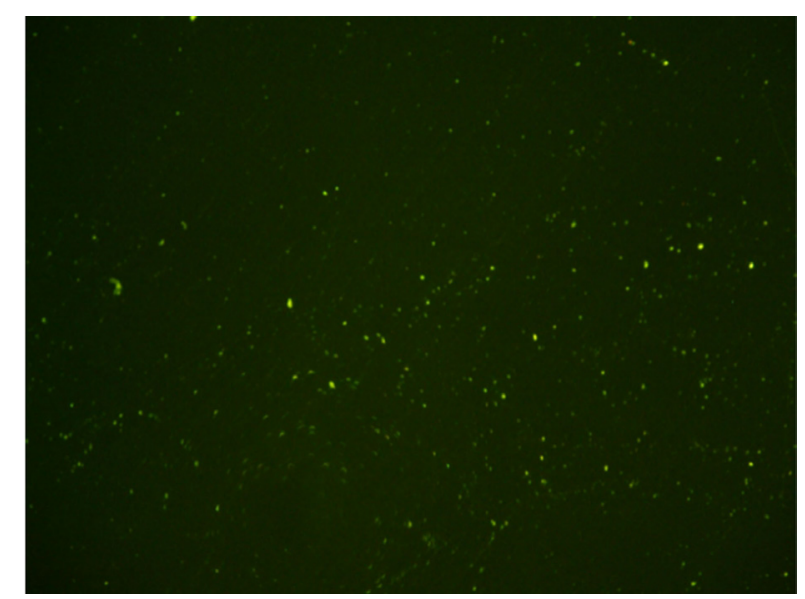

Figure 5. NSCs marked with BrdU.

\section{Identification of complex of MBL and NSCs}

Sections showed NSCs were evenly distributed in MBL. The complex contained the most cells on the 5th day after transplantation and began to reduce on the 7th day (Figures $6 \mathrm{~A}-\mathrm{C}$ and $7 \mathrm{~A}-\mathrm{B})$.
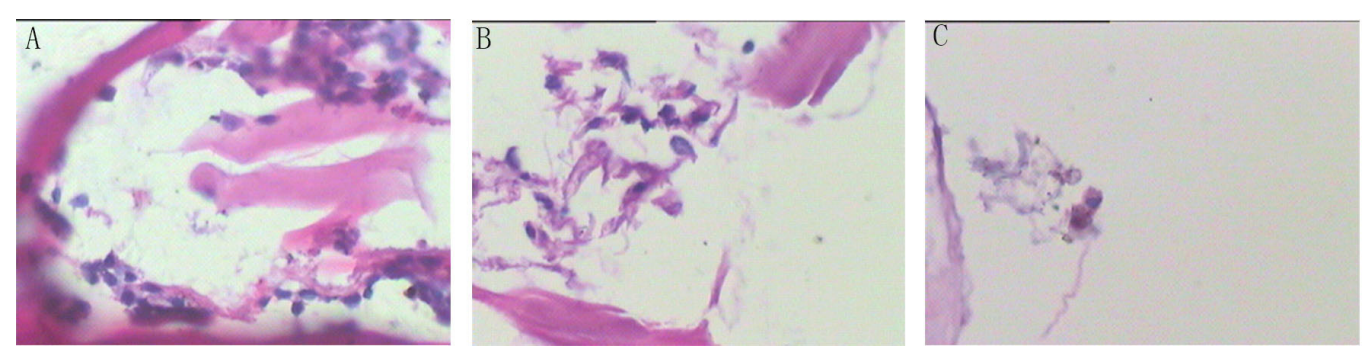

Figure 6. NSCs in the MBL. Results for hematoxylin and eosin staining have been provided. A. Three days after translation. B. Five days after translation. C. Seven days after translation.

\section{Evaluation of complex of MBL and OECs}

When cultivated for 5 days, OECs were evenly distributed in the MBL. The density of OECs on the 7th day was higher than on the 5th day, and no additional changes were observed on the 9th day (Figure 8). 

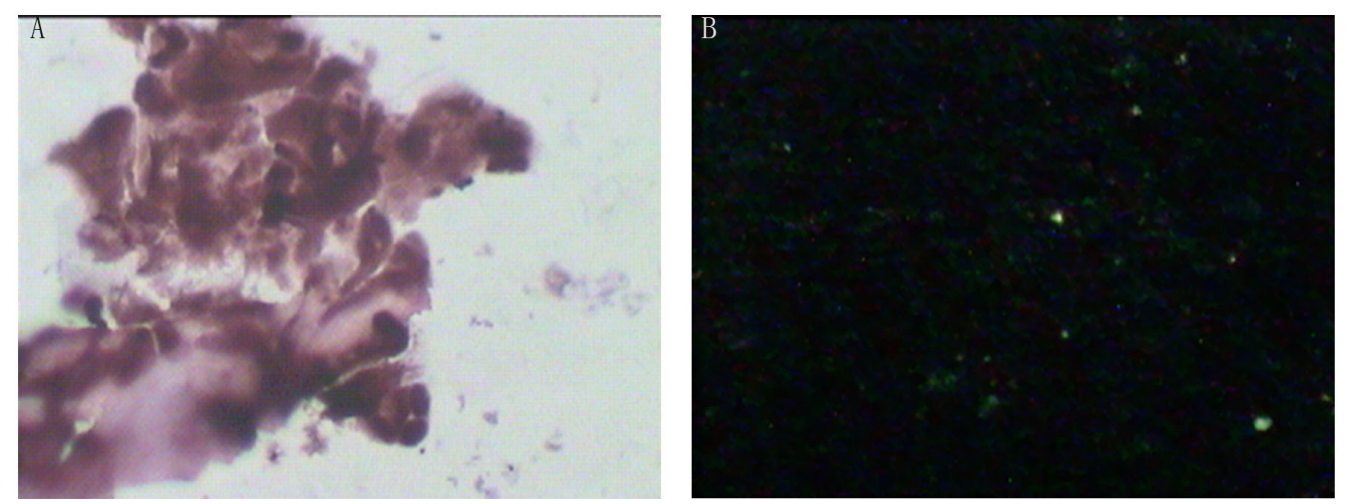

Figure 7. NSCs in the MBL. A. Immunohistochemistry (40X). B. Immunofluorescence (40X).

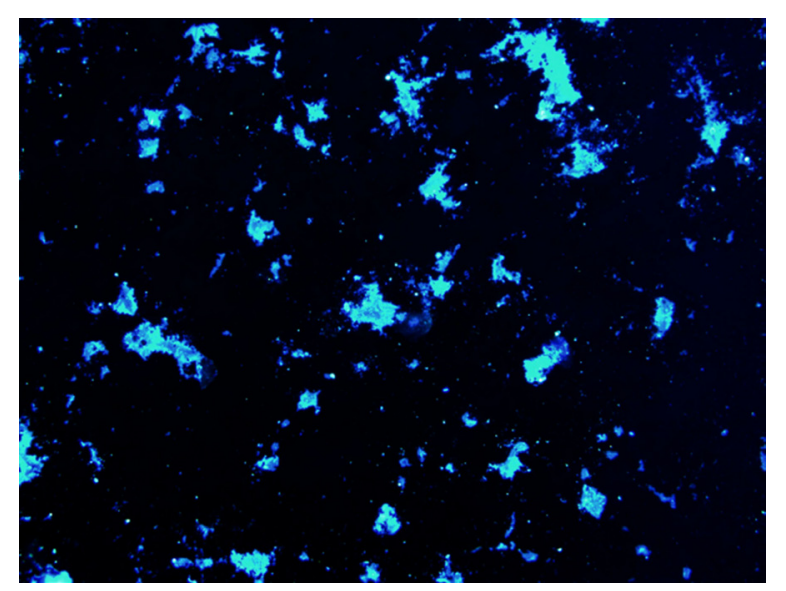

Figure 8. OECs in the MBL identified by immunofluorescence (40X; excited by ultraviolet light).

\section{Examination of complex of NECs, OECs, and MBL}

Immunofluorescence microscopy after cultured for 5 days showed that NSCs with green fluorescence and OECs with blue fluorescence densely covered the MBL as viewed under ultraviolet radiation (Figure 9).

\section{Morphological changes observed after operation}

In Group A, fibrillar connective tissues proliferated together with injured tissue death and cavity formation (Figure 10). In Group B, we observed a relatively larger cavity at the injury site, few NSCs, a severe inflammatory response, obvious inflammatory cell infiltration, and numerous fibrous scars (Figure 11) at the 4th week. In Group C, positively stained nerve fiber cells were scarce and irregularly arranged; inflammatory cell infiltration was also severe (Figure 12). In Group D, immunofluorescence was tested at the 4th week and NSCs were 
found to have migrated and distributed from the cranial to the caudal site (Figure 13A-B). Immunohistochemical results showed that transplanted cells in the MBL had also moved to the caudal side, and host cells had grown in the MBL. In addition, we observed grey and white matter with clear boundaries at the lacerated ends, the nerve fibers were organized, the areas were free of necrosis, the cystic cavity had significantly narrowed, and the transplanted cells had fused together with normal tissue around them (Figure 14A-B). In Group E, immunohistochemistry and immunofluorescence results at the 4th week revealed that OECs had formed myelin sheath around nerve fiber synapses and could pass through the transplanted areas. Few inflammatory cells and no obvious inflammatory infiltration were observed (Figure 15A-B). In Group F, pathological sections at the 4th week showed fiber scars and sporadic inflammatory cells, polygonal cells, and small round cells at the lacerated ends, as well as BrdU-positive NSCs and P75-positive OECs as shown by immunohistochemistry and immunofluorescence double staining (Figures 16 and 17). Additionally, immunohistochemical double staining of NSE and BrdU indicated that some of the double-positive cells were neurons that had transformed from BrdU-labeled neural stem cells (Figure 18).

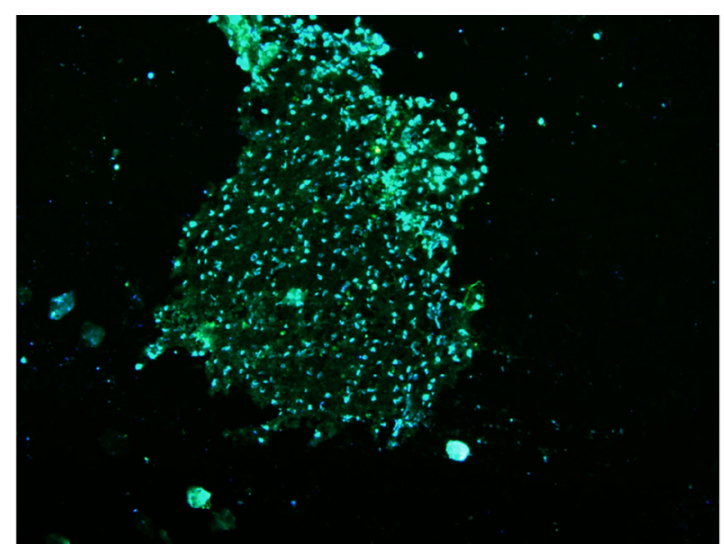

Figure 9. Identification of NSCs + OECs in the MBL using immunofluorescence (10X; excited by ultraviolet light).

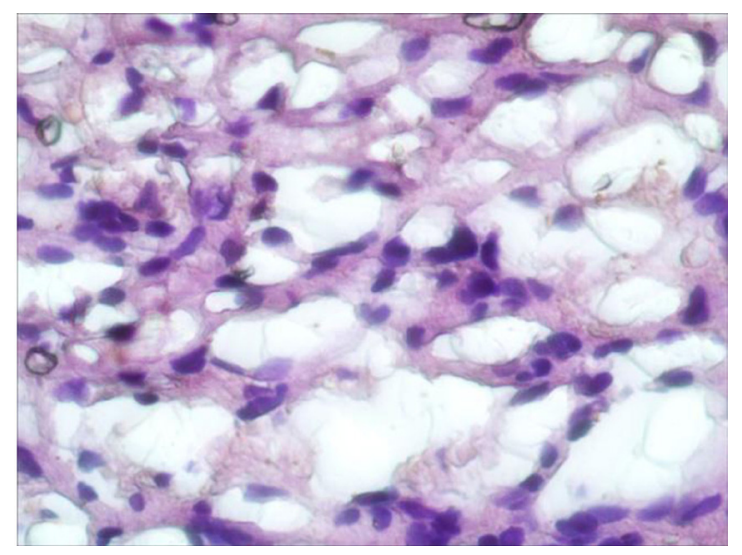

Figure 10. Group A, 4 weeks after surgery (immunohistochemistry, 40X). 


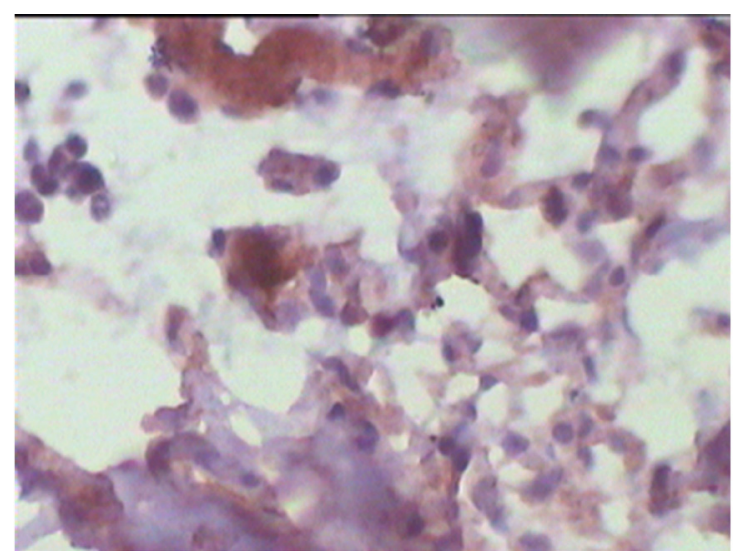

Figure 11. Group B, 4 weeks after surgery (immunohistochemistry, 40X).

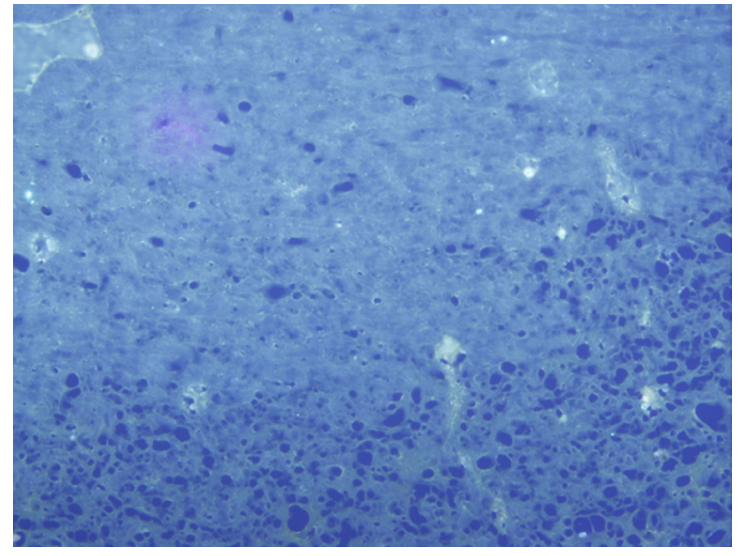

Figure 12. Group C, 4 weeks after surgery (immunofluorescence, 10X, excited by ultraviolet light).
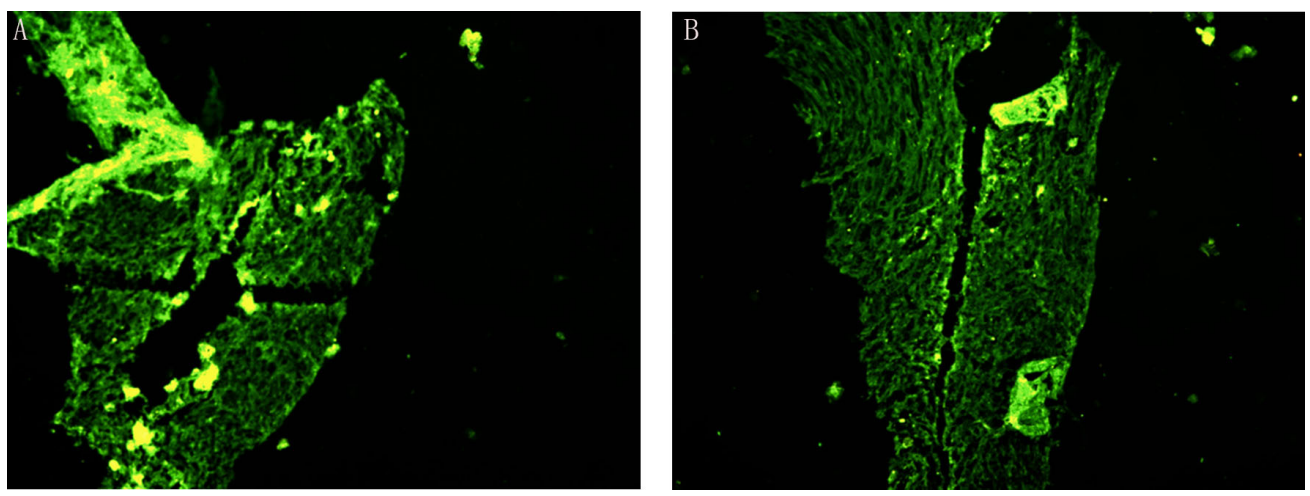

Figure 13. Group D, 4 weeks after surgery (immunofluorescence, excited by ultraviolet light). A. Cross section (40X). B. Vertical section (40X). 

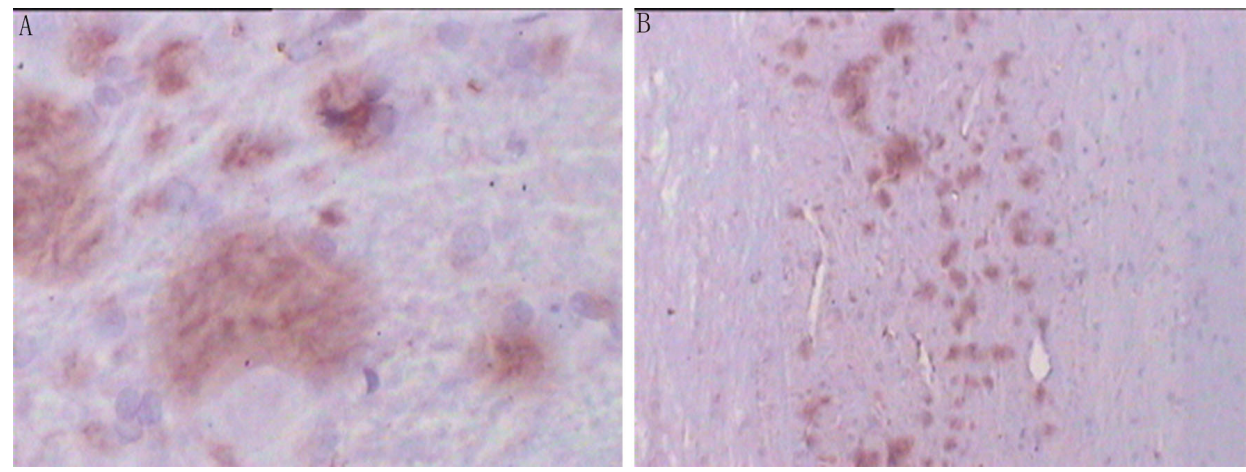

Figure 14. Group D, 4 weeks after surgery (immunohistochemistry, 40X). A. Cross section (40X). B. Vertical section (40X).
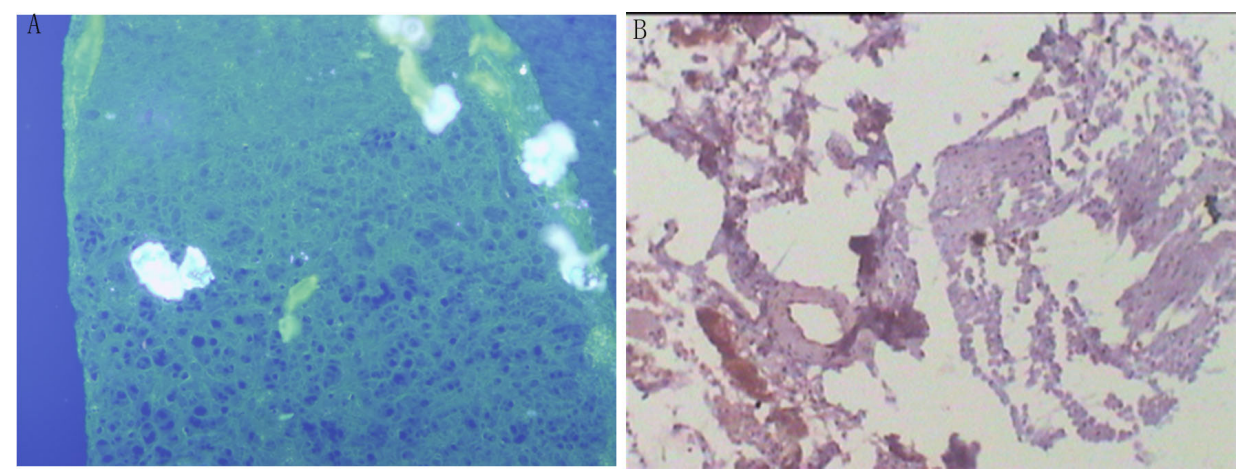

Figure 15. Group E, 4 weeks after surgery (40X). A. Immunofluorescence. B. Immunohistochemistry.

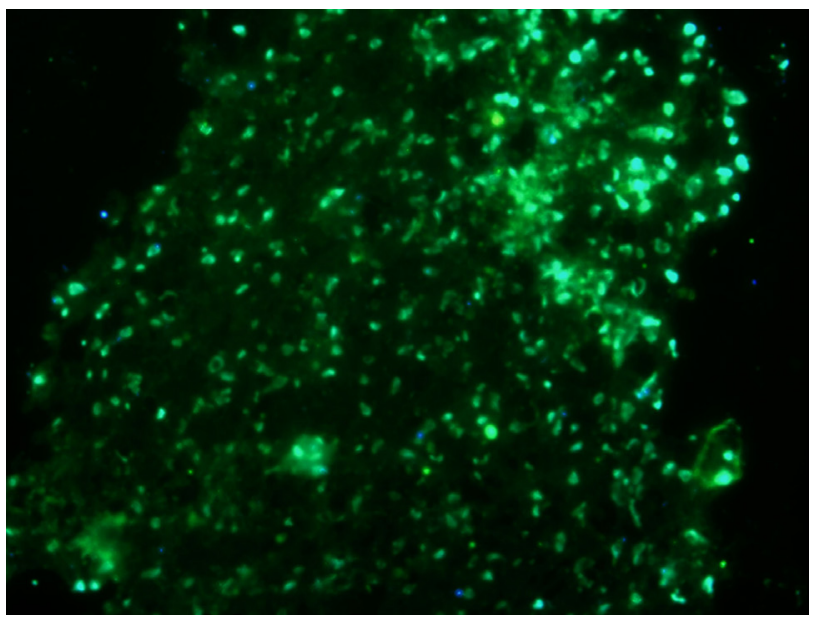

Figure 16. Group F, 4 weeks after surgery (immunofluorescence, 10X; OECs excited by ultraviolet are blue, NSCs are green). 


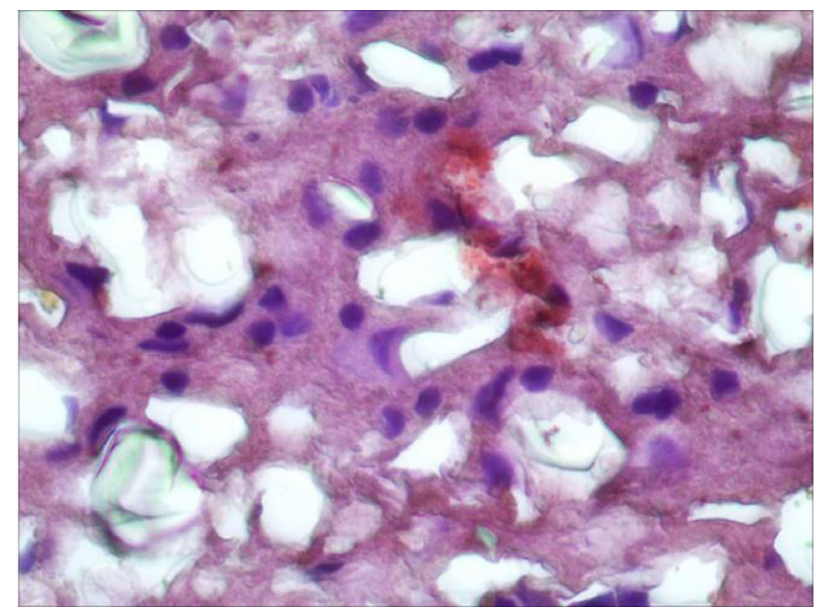

Figure 17. Group F immunohistochemical double staining. BrdU+ cells are black, and P75+ cells are red.

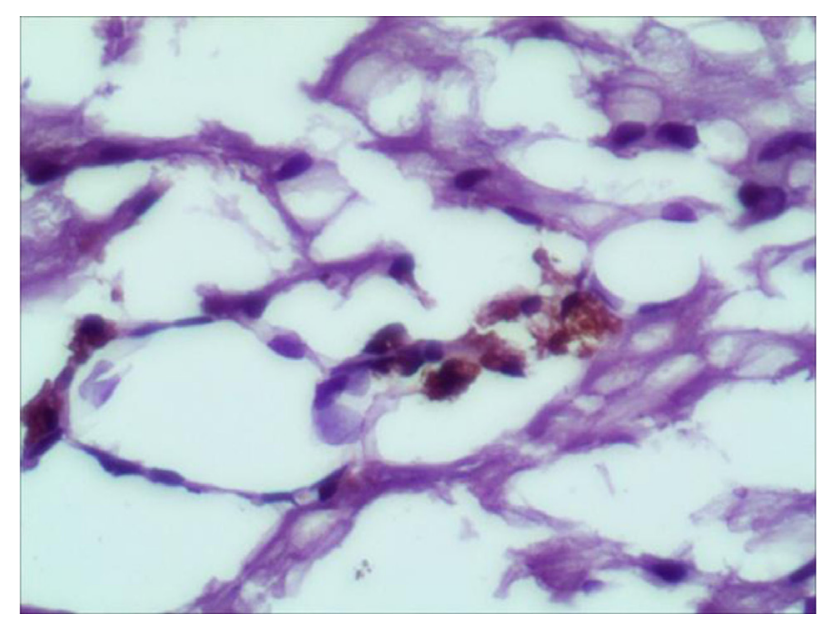

Figure 18. Group F immunohistochemical double staining. BrdU+ cells are black, and NSE + cells are red.

\section{Electron microscopy results of post-surgery samples}

In Group F, MBL in the site of injury was not autolyzed based on scanning electron microscopy results at the 4th week, and they densely covered the site of injury in a honeycomb pattern (Figure 19A). New neurons and their axons were found to be embedded in the connective area of the MBL and spinal cord; moreover, nerve fiber bundles passed through this area (Figure 19B). Transmission electron microscopy showed that neurons containing Nissl bodies were found as well as synapses and axons; however, demyelination of the outer layers of the axons was also detected (Figure 20A-C). 

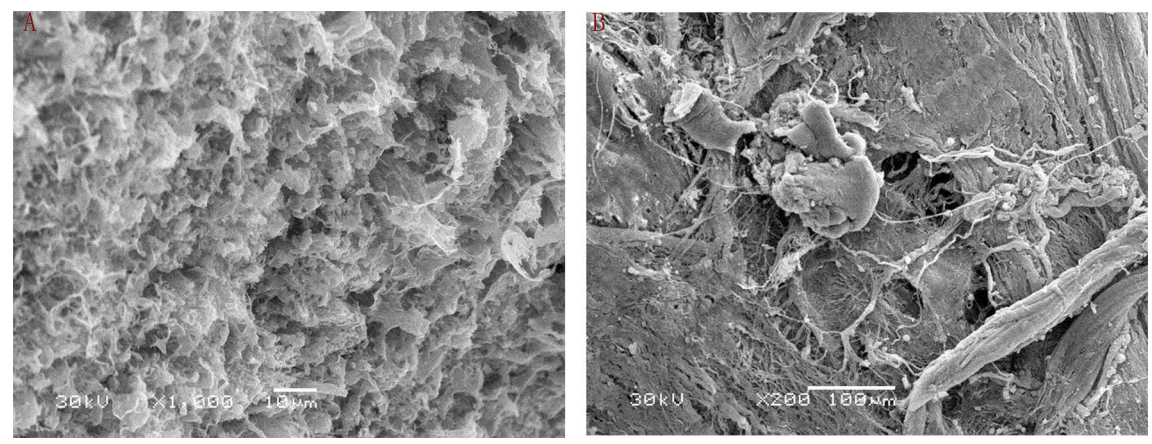

Figure 19. Scanning electron microscopy images of group F 4 weeks after surgery.
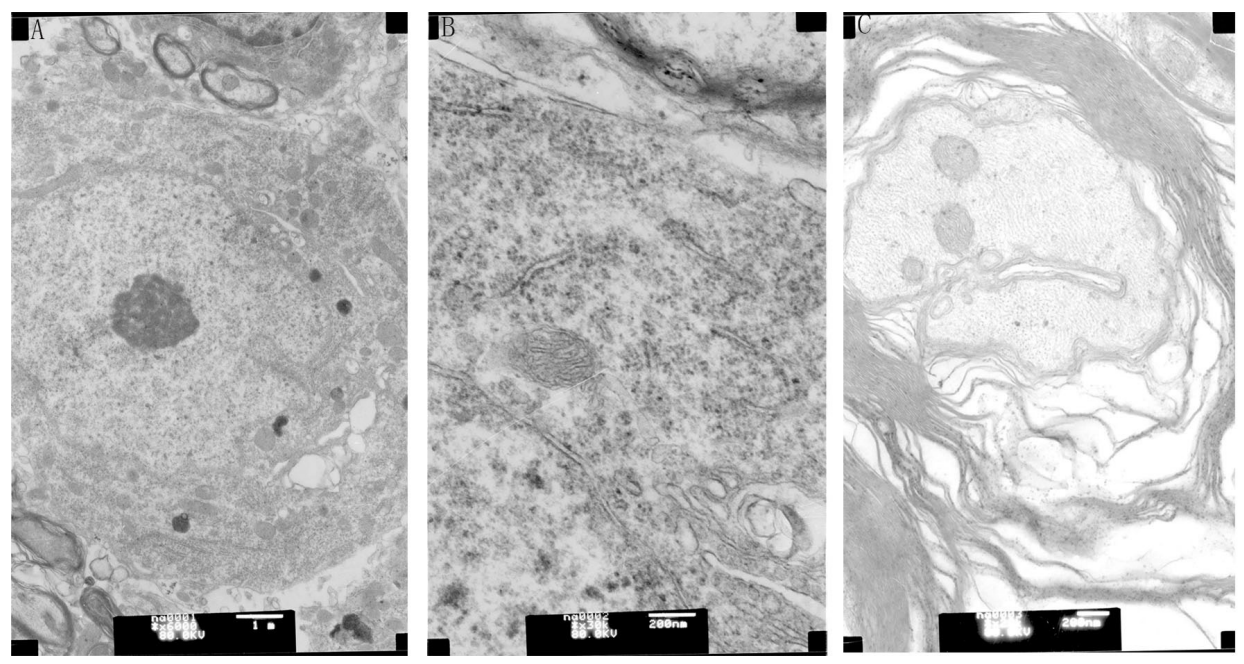

Figure 20. TEM images of group F 4 weeks after surgery.

\section{Locomotor function assessment after transplantation and statistical analysis}

Before surgery, rats in each group were scored 21 according to BBB. Three days after surgery, 1 rat from group A died of urinary tract infections and thus was not analyzed. We performed statistical analysis on the data of remaining rats. Details are shown in Tables 1 and 2.

Table 1. BBB scores of 6 groups at each point after surgery (means \pm SD, point).

\begin{tabular}{lcccr}
\hline & 1 day before surgery & 2 weeks after surgery & 4 weeks after surgery & 8 weeks after surgery \\
\hline A & 21 & $3.45 \pm 0.312$ & $3.82 \pm 0.226$ & $3.71 \pm 0.286$ \\
B & 21 & $4.33 \pm 0.310$ & $6.25 \pm 0.305$ & $8.25 \pm 0.648$ \\
C & 21 & $3.75 \pm 0.250$ & $5.17 \pm 0.345$ & $7.13 \pm 0.479$ \\
D & 21 & $4.50 \pm 0.261$ & $7.92 \pm 0.288$ & $11.38 \pm 0.532$ \\
E & 21 & $4.33 \pm 0.414$ & $6.83 \pm 0.345$ & $10.63 \pm 0.565$ \\
F & 21 & $4.58 \pm 0.379$ & $8.83 \pm 0.613$ & $11.86 \pm 0.738$ \\
\hline
\end{tabular}




\begin{tabular}{|c|c|c|c|c|c|c|}
\hline \multirow[t]{2}{*}{ Group } & \multicolumn{2}{|c|}{2 weeks after surgery } & \multicolumn{2}{|c|}{4 weeks after surgery } & \multicolumn{2}{|c|}{8 weeks after surgery } \\
\hline & t point & P point & t point & $\overline{\text { P point }}$ & t point & P point \\
\hline$A \& B$ & -1.995 & 0.059 & -6.313 & 0.000 & -6.089 & 0.000 \\
\hline$A \& C$ & -0.745 & 0.465 & -3.207 & 0.004 & -5.885 & 0.000 \\
\hline$A \& D$ & -2.584 & 0.017 & -11.059 & 0.000 & -12.147 & 0.000 \\
\hline$A \& E$ & -1.669 & 0.110 & -7.172 & 0.000 & -10.431 & 0.000 \\
\hline$A \& F$ & -2.276 & 0.033 & -7.404 & 0.000 & -10.293 & 0.000 \\
\hline$B \& D$ & -0.411 & 0.685 & -3.978 & 0.001 & -3.727 & 0.002 \\
\hline$C \& E$ & -1.205 & 0.241 & -3.421 & 0.002 & -4.723 & 0.000 \\
\hline$D \& E$ & 0.340 & 0.737 & 2.414 & 0.025 & 0.966 & 0.350 \\
\hline $\mathrm{D} \& \mathrm{~F}$ & -0.181 & 0.858 & -1.353 & 0.190 & -0.540 & 0.599 \\
\hline $\mathrm{E} \& \mathrm{~F}$ & -0.445 & 0.660 & -2.843 & 0.009 & -1.345 & 0.202 \\
\hline
\end{tabular}

\section{DISCUSSION}

Recent pathological studies have suggested that the difficulties of functional recovery after SCI mainly included the survival of cells, regeneration of axons, the precise location of regenerative axons, and accurate reformation and functional synaptic connections (Nandoe Tewarie et al., 2009). Because NSCs have the potential to differentiate into neurons (Lee et al., 2012), oligodendrocytes, and axons (Decimo et al., 2011; Yasuda et al., 2011) and also have the ability to fill the spinal cord cavity and blend with host spinal cord tissues (Boido et al., 2011; Li et al., 2011), they may be useful for repairing SCI. Animal experiments have indicated that the regenerated axon has limited ability to cross fiber scars to set up new neural pathways because the internal environment of the organism affects the survival and differentiation of NSCs (Lian Jin et al., 2011; Meng et al., 2011; Su et al., 2011; Wang et al., 2011; Wakai et al., 2014). Therefore, limited effects would be observed for the repair using only NSCs. Thus, 2 critical issues should be addressed to improve the effectiveness of NSC transplantation for SCI repair. First, a passage location for axon growth should be provided (Amr et al., 2014; Binan et al., 2014; Pera et al., 2014); second, a stable microenvironment rich in nutrients and nerve growth factors should be ensured (Paczkowska at al., 2013; Uesaka et al., 2013; Ladewig et al., 2014; Shin et al., 2014). MBL is a new scaffold material and is a natural biomaterial made by removing muscle cells, panniculus adiposus, membrane-associated antigens, and soluble protein; MBL is similar to the neural membrane tube. It shows good biocompatibility with nervous tissue as well as good contact orientation function, which addresses the first limitation described before. However, studies reported that transplantation of MBL alone was autolysisprone, while transplanting MBL injected with nerve growth factors avoided autolysis (Krenz and Weaver, 2000). OEC is a special glial cell existing in the olfactory epithelium and olfactory nerve (Ekberg and St. John, 2014). It can develop both in the peripheral nervous system and central nervous system and shares functions with Schwann cell and astrocytes (Montgomery et al., 1996). OEC can secrete brain-derived neurotrophic factor and glial cell line-derived neurotrophic factor, among others, which can facilitate the regeneration of synapses ( $\mathrm{Lu}$ and Waite, 1999; Fairless and Barnett, 2005; King-Robson, 2011; Kerever at al., 2014; Li et al., 2014). In addition, cell adhesion molecules and adhesive glycoproteins are secreted from OECs and have a positive effect on axon growth and tunica vaginalis formation outside of axons; OECs also inhibit hyperplasia of glial cells, the formation of scars, and reduce the loss of tissues at the injury site (Chuah et al., 2011). Therefore, as an ideal cell for transplantation, OECs not only overcome the second limitation, but also prevent MBL from autolyzing. 
In this study, we transplanted MBL carrying NSCs and OECs to the lacerated ends of the injured spinal cord to treat SCI (Group F). We also compared the effectiveness of this method with other methods transplanting NSCs only (group B), OECs only (group C), NSCs + MBL (group D), and OECs + MBL (group E).

Pathological sections of group B (transplantation with NSCs only) and C (transplantation with OECs only) samples indicated that only a few transplanted cells were still alive in the grafted area and that there were severe inflammation responses. No regenerated axons crossed the scar barriers. This reflects limited repair function for these 2 groups. However, in groups $\mathrm{D}, \mathrm{E}$, and $\mathrm{F}$ using MBL, a larger number of grafted cells were observed and were alive than in groups $\mathrm{B}$ and $\mathrm{C}$. In addition, pathological sections also revealed that MBL may be a barrier to inflammatory cells to some extent, which would in turn reduce the harm to grafted cells caused by inflammatory cells and enable formation of the connection between transplanted cells and host cells. This may explain why transplantation with MBL improved the recovery of SCI in rats. Additionally, our results were consistent with BBB scoring data. Grafting with MBL did not lead to an apparent inflammation reaction because the antigen components of MBL had been removed, providing good histocompatibility.

Examination of the pathological sections in group D and E showed that NSCs in the MBL migrate from the lacerated ends to the caudal region and were integrated into the host spinal cord tissues. A larger number of living NSCs were detected compared to when only NSCs were grafted, inflammatory reactions were less severe, and scars did not cut off the basal membrane tube. These outcomes demonstrate MBL can not only facilitate the oriented growth of NSCs along its lumen, but also help host cells migrate along extracellular matrix within its lumen. These cells act as a bridge to promote nerve cell integration from the 2 lacerated ends and form synaptic connections. Fansa et al. (2002) suggested that extracellular matrix-like fibronectin and laminin remain in the MBL and play a key role in cell adherence and migration. More specifically, laminin can guide and facilitate axon growth into the lumen, while fibronectin promotes the regeneration of axons and prevents over-infiltration of fibroblasts. Similarly, group E showed slight inflammatory responses and cell infiltration. Some host nerve cells were also found to have migrated into the lumen across the scars. This may be because MBL can create a microenvironment, which would ensure that brain-derived neurotrophic factor and glial cell line-derived neurotrophic factor secreted by OECs were present at a relatively high concentration to better facilitate nerve growth. Additionally, BBB scoring in the 4th week also revealed that rats in groups $\mathrm{D}$ and $\mathrm{E}$ showed better recovery in hind limb motor function. However, rats in group $\mathrm{D}$ showed even better performance compared with rats in group E; the difference was significant. This may be because there were relatively smaller absolute amounts of nerve cells connecting within the MBL in group E, which has some disadvantages regarding the recovery of neural pathways between the downlink and the uplink.

In group F, the results of morphological analysis revealed living NSCs and OECs within the MBL and that some neural stem cells had transformed into neurons. These neurons in the MBL had already issued axons along the lumen according to electron microscopy results. Additionally, regenerative synaptic connections were observed, but we also observed demyelination in the out layer of these axons. This indicated that OECs play a limited role in myelin sheath formation and that the regenerative axons were not sufficiently healthy. This shortage may be an important factor affecting signal transmittance between nerves. Consistent 
with this, the results of BBB scoring showed that rats in this group clearly had better hind limb function compared with rats in groups A, B, C, and E; however, compared with group D, there was no significant difference despite the higher score. This may be because of several factors. First, some NSCs located in the MBL were transformed into neurons, so there were more nerve cells in the lumen compared with in group $\mathrm{E}$, which was beneficial for generating more axons and synaptic connections as well as for establishing more nerve pathways. Hence, group E showed better recovery of SCI than did group E. However, demyelination of these regenerative axons was observed, which would greatly restrict the transmittance of nerve signals. Additionally, it remains unclear whether regenerative axon synaptic connections contain effective axons that can transmit nerve signals. This may have affected the effectiveness of the newly formed nerve pathways. Finally, although there were OECs to help form myelin sheath and to nourish nerves in the MBL, which might have a role on neural stem cells inducing, axon growth and synaptic connections, these functions were limited. These 3 factors may together have affected our results; the BBB score of group F was slightly higher than that in group D, but the difference was not significant.

In conclusion, transplantation with MBL carrying NSCs and OECs as well as MBL carrying NSCs may improve the recovery of rat neurologic function after SCI. However, the outcome is still far from ideal. The loading capability of rat hind limbs and motor coordination ability of the hind and fore limbs were not sufficiently recovered. In addition, there were no substantial differences between the 2 methods tested. This may be because the grafted NSCs and OECs could not function together to a great extent. Therefore, identifying an efficient pathway for transmitting signals between these cell types, preventing demyelination, and establishing effective synaptic connections may be the key points in overcoming these limitations, similar to the approach discussed for the Notch pathway and Sox2 (Hoffmann et al., 2014; Kim et al., 2014; Koike et al., 2014; Kotasová et al., 2014; Kim et al., 2014; Liu et al., 2014). Additional studies are needed to clarify these points.

\section{Conflicts of interest}

All the authors declare no conflict of interest.

\section{ACKNOWLEDGMENTS}

Research supported by the Second Hospital of Lanzhou University. We thank Kang $\mathrm{X}$.W., as the corresponding author, for guidance and assistance and the supporting of teachers in Lanzhou University. The sectional chief and the teachers in the Department of Orthopedics in Second Hospital of Lanzhou University supported our experimental expenditure.

\section{REFERENCES}

Amr SM, Gouda A, Koptan WT, Galal AA, et al. (2014). Bridging defects in chronic spinal cord injury using peripheral nerve grafts combined with a chitosan-laminin scaffold and enhancing regeneration through them by co-transplantation with bone-marrow-derived mesenchymal stem cells: case series of 14 patients. J. Spinal Cord Med. 37: 54-71.

Binan L, Tendey C, De Crescenzo G, El Avoubi R, et al. (2014). Differentiation of neuronal stem cells into motor neurons using electrospun poly-L-lactic acid/gelatin scaffold. Biomaterials 35: 664-674.

Boido M, Garbossa D and Vercelli A (2011). Early graft of neural precursors in spinal cord compression reduces glial cyst 
and improves function. J. Neurosurg. Spine 15: 97-106.

Chuah MI, Hale DM and West AK (2011). Interaction of olfactory ensheathing cells with other cell types in vitro and after transplantation: glial scars and inflammation. Exp. Neurol. 229: 46-53.

Decimo I, Bifari F, Rodriguez FJ, Malpeli G, et al. (2011). Nestin- and doublecortin-positive cells reside in adult spinal cord meninges and participate in injury-induced parenchymal reaction. Stem Cells 29: 2062-2076.

Ekberg JA and St. John JA (2014). Crucial roles for olfactory ensheathing cells and olfactory mucosal cells in the repair of damaged neural tracts. Anat. Rec. 297: 121-128.

Fairless R and Barnett SC (2005). Olfactory ensheathing cells: their role in central nervous system repair. Int. J. Biochem. Cell Biol. 37: 693-699.

Fansa H, Schneider W, Wolf G and Keilhoff G (2002). Host responses after acellular muscle basal lamina allografting used as a matrix for tissue engineered nerve grafts1. Transplantation 74: 381-387.

Hoffmann SA, Hos D, Kuspert M, Lang RA, et al. (2014). Stem cell factor Sox2 and its close relative Sox3 have differentiation functions in oligodendrocytes. Development 141: 39-50.

Kerever A, Mercier F, Nonaka R, de Vega S, et al. (2014). Perlecan is required for FGF-2 signaling in the neural stem cell niche. Stem Cell Res. 12: 492-505.

Kim EY, Lee KB, Yu J, Lee JH, et al. (2014). Neuronal cell differentiation of mesenchymal stem cells originating from canine amniotic fluid. Hum. Cell 27: 51-58.

Kim HS, Kim J, Jo Y, Jeon D, et al. (2014). Direct lineage reprogramming of mouse fibroblasts to functional midbrain dopaminergic neuronal progenitors. Stem Cell Res. 12: 60-68.

King-Robson J (2011). Encouraging regeneration in the central nervous system: is there a role for olfactory ensheathing cells? Neurosci. Res. 69: 263-275.

Koike T, Wakabayashi T, Mori T, Takamori Y, et al. (2014). Sox2 in the adult rat sensory nervous system. Histochem. Cell Biol. 141: 301-309.

Kotasová H, Procházková J and Pacherník J (2014). Interaction of Notch and gp130 signaling in the maintenance of neural stem and progenitor cells. Cell Mol. Neurobiol. 34: 1-15.

Krenz NR and Weaver LC (2000). Nerve growth factor in glia and inflammatory cells of the injured rat spinal cord. $J$. Neurochem. 74: 730-739.

Ladewig J, Koch P and Brüstle O (2014). Auto-attraction of neural precursors and their neuronal progeny impairs neuronal migration. Nat. Neurosci. 17: 24-26.

Lee KB, Choi JH, Byun K, Chung KH, et al. (2012). Recovery of CNS pathway innervating the sciatic nerve following transplantation of human neural stem cells in rat spinal cord injury. Cell Mol. Neurobiol. 32: 149-157.

Li Y, Zhang WM and Wang TH (2011). Optimal location and time for neural stem cell transplantation into transected rat spinal cord. Cell Mol. Neurobiol. 31: 407-414.

Li YC, Tsai LK, Wang JH and Young TH (2014). A neural stem/precursor cell monolayer for neural tissue engineering. Biomaterials 35: 1192-1204.

Lian Jin H, Pennant WA, Hyung Lee M, Su S, et al. (2011). Neural stem cells modified by a hypoxia-inducible VEGF gene expression system improve cell viability under hypoxic conditions and spinal cord injury. Spine 36: 857-864.

Liu Q, Fan X, Zhu J, Xu G, et al. (2014). Co-culturing improves the OGD-injured neuron repairing and NSCs differentiation via Notch pathway activation. Neurosci. Lett. 559: 1-6.

Lu J and Waite P (1999). Advances in spinal cord regeneration. Spine 24: 926-930.

Meng QQ, Liang XJ, Wang P, Wang XP, et al. (2011). Rosiglitazone enhances the proliferation of neural progenitor cells and inhibits inflammation response after spinal cord injury. Neurosci. Lett. 503: 191-195.

Montgomery CT, Tenaglia EA and Robson JA (1996). Axonal growth into tubes implanted within lesions in the spinal cords of adult rats. Exp. Neurol. 137: 277-290.

Nandoe Tewarie RS, Hurtado A, Bartels RH, Grotenhuis A, et al. (2009). Stem cell-based therapies for spinal cord injury. J. Spinal Cord. Med. 32: 105-114.

Paczkowska E, Kaczynska K, Pius-Sadowska E, Rogińska D, et al. (2013). Humoral activity of cord blood-derived stem/ progenitor cells: implications for stem cell-based adjuvant therapy of neurodegenerative disorders. PLoS One 8: e83833.

Pera EM, Acosta H, Gouignard N, Climent M, et al. (2014). Active signals, gradient formation and regional specificity in neural induction. Exp. Cell Res. 321: 25-31.

Shin DA, Pennant WA, Yoon DH, Ha Y, et al. (2014). Co-transplantation of bone marrow-derived mesenchymal stem cells and nanospheres containing FGF-2 improve cell survival and neurological function in the injured rat spinal cord. Acta Neurochir. 156: 297-303.

Su H, Wu Y, Yuan Q, Guo J, et al. (2011). Optimal time point for neuronal generation of transplanted neural progenitor 
cells in injured spinal cord following root avulsion. Cell Transplant. 20: 167-176.

Uesaka T, Nagashimada M and Enomoto H (2013). GDNF signaling levels control migration and neuronal differentiation of enteric ganglion precursors. J. Neurosci. 33: 16372-16382.

Wakai T, Sakata H, Narasimhan P, Yoshioka H, et al. (2014). Transplantation of neural stem cells that overexpress SOD1 enhances amelioration of intracerebral hemorrhage in mice. J. Cereb. Blood Flow Metab. 34: 441-449.

Wang JM, Zeng YS, Wu JL, Li Y, et al. (2011). Cograft of neural stem cells and schwann cells overexpressing TrkC and neurotrophin-3 respectively after rat spinal cord transection. Biomaterials 32: 7454-7468.

Yasuda A, Tsuji O, Shibata S, Nori S, et al. (2011). Significance of remyelination by neural stem/progenitor cells transplanted into the injured spinal cord. Stem Cells 29: 1983-1994. 